\title{
Modeling of Open Square Bifilar Planar Spiral Coils
}

\author{
Denivaldo P. da Silva ${ }^{1}$ and Sérgio F. Pichorim ${ }^{2}$ \\ Graduate School of Electrical Engineering and Applied Computer Sciences (CPGEI) \\ Federal University of Technology - Paraná (UTFPR), Curitiba-PR, Brazil \\ denivaldo.silva@ifsc.edu.br ${ }^{1}$,pichorim@utfpr.edu.br ${ }^{2}$,
}

\begin{abstract}
In this paper an electrical model for square bifilar planar spiral coils (BPSC) is presented. Its main aim is the study of BPSC electrical parameters and behavior involving the frequency range where the first resonances (valley and peak) occur for bifilar coils in open-circuit configuration. A new approach to determine mutual capacitances of BPSCs based on coplanar waveguide (CPW) lines is presented. This study can be applied for modeling of passive self-resonant (PSR) sensors and wireless power transfer (WPT) systems. In order to validate the proposed model, three BPSCs were manufactured, tested by means of an impedance analyzer and also submitted to electromagnetic (EM) simulations. The results obtained, presented by means of tables and graphs, show that the present study is feasible and promising for the modeling of open square BPSCs.
\end{abstract}

Index Terms - Bifilar Coil, Electrical Modeling, Planar Spiral Coil, SelfResonance.

\section{INTRODUCTION}

The conception of bifilar winding dates back to the last decade of the nineteenth century and is related to studies of the Serbian engineer Nikola Tesla (1856-1943) in the development of electrical devices intended to transmit and distribute high frequency electrical energy [1]-[2].

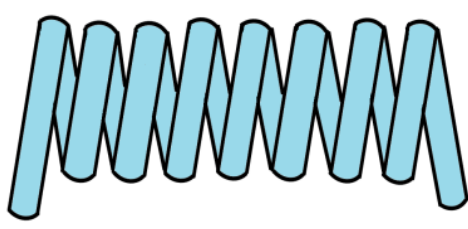

(a)

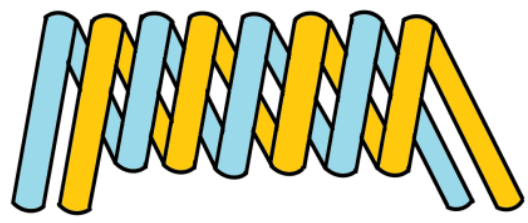

(b)

Fig. 1. Helical monofilar (a) and bifilar (b) coils

As an example, Fig. 1 shows the difference between the winding method of a monofilar and bifilar coil for the helical shape. According to [3], considering both coils of the same shape, diameter, wire spacing and same turn number, the mutual capacitance arising between bifilar coil windings is significantly greater than the self-capacitance [4] arising in the terminals of the monofilar coil. This is due to fact that the average voltage between adjacent turns in the bifilar coil is greater than in the monofilar coil by a proportionality ratio that is function of the number of turns [3], [5]. Thus, the higher the number of turns, the higher the rate of proportionality between these two capacitances. Consequently, this makes the first self-resonance frequency of the bifilar coil significantly smaller 
than the one of a monofilar coil. This is an advantage, for example, in biomedical applications where the signal received by a reader coil from an implanted sensor in a biological tissue tends to be less attenuated with frequency reduction [6].

In addition to the helical shape [3], [5], [7] the bifilar coil also may have Archimedean planar spiral [8], square planar spiral [9], [10], hexagonal planar spiral or even octagonal planar spiral shapes [11]. However, this paper is limited only to the study of the square bifilar planar spiral coil (BPSC).

When manufactured on printed circuit board (PCB), square BPSCs may present lower manufacturing complexity than hexagonal, octagonal and, in particular, Archimedean BPSCs because their tracks maintain angles of 90 degrees between them [12].

BPSC can be applied, for example, as a passive self-resonant (PSR) sensor in the monitoring of soil [9] and wood [10] moisture, as well as for monitoring pressure, force and displacement [11]. This monitoring is done, indirectly, by successive measurements of parallel resonance frequencies, where the first impedance peak occurs. These variations in the resonance frequency are due to the variations suffered by the physical quantity monitored.

PSR sensor resonates at a certain self-resonant frequency without the aid of external capacitors due to the inductive and capacitive effect that occurs in their metal tracks and the influence of the medium that surrounds them. Generally, they are small, in the order of a few tens of millimeters, manufactured in PCB and coated with solder mask to protect against corrosion and short circuits between copper tracks.

At low frequencies and disregarding resistive losses, the bifilar coil can be modeled, in the opencircuit and closed-circuit configurations, as being two monofilar coils B1 and B2 with terminal pairs (1)-(2) and (3)-(4) and with their self-inductances $L_{s 1}$ and $L_{s 2}$ that are magnetically coupled with a mutual inductance $M$, as shows Fig.2.

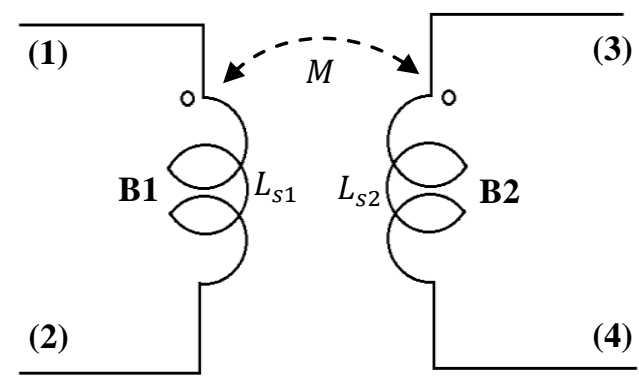

(a)

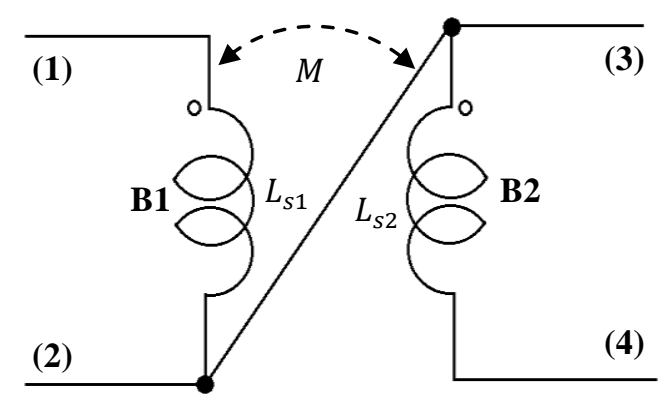

(b)

Fig. 2. Simplified lossless electrical models of a bifilar coil in the open-circuit configuration (a) and in the closed-circuit configuration (b).

In its 1894 patent, Tesla has studied only the closed-circuit configuration of its bifilar coil as in Fig. 2(b), but this coil can also be studied in the open-circuit configuration without the jumper between terminals (2)-(3), as shown in Fig. 2(a). However, regardless of the studied configuration (open or closed) the bifilar coil is generally analyzed by its terminals (1)-(4). 
The current studies on square BPSC are generally still restricted to closed-circuit configuration for application as PSR sensor as in [9], [10]. However, in open-circuit configuration, two BPSCs can be applied also for wireless power transfer (WPT) through their series resonances where the first impedance valley occurs, just as in [7] where tests with helical coils were performed.

Impedance curve $Z_{14}$ of the open BPSC can be obtained by an impedance analyzer, but for the design of BPSC, it is interesting to predict it by means of an electric model that can determine with accuracy the first valley and the first peak of resonance.

Studies on modeling of open BPSCs acting at the series and parallel resonances are still rare. In [11] an open square BPSC is shown, but an electric model is not provided. In [8] the open BPSC is presented in the Archimedean shape, but the authors adopted an ideal electric model that covers only one resonance frequency and with an error of $22 \%$ relative to measured data.

This paper presents an electric model for the open square BPSC that covers the first valley and the first peak of resonance. The electrical parameters of this model were obtained and a new approach to determine mutual capacitances of BPSCs, based on coplanar waveguide (CPW) lines, is presented. In order to validate the proposed model, three square BPSCs were manufactured in double-sided PCB with FR-4 substrate and coated with solder mask. These BPSCs were tested by an impedance analyzer and also submitted to electromagnetic (EM) simulations. Tables and graphics were produced aiming to establish a comparative analysis between the results obtained with the proposed model, by EM simulation, as well as for the measured values.

\section{MODELING OF OPEN SQUARE BPSC}

Fig. 3 shows, as an example, an open square BPSC with $N=4$ turns, formed by two monofilar planar spiral coils (PSC), each one with $N_{\mathrm{m}}=2$ turns, where $w$ is the width of the copper track and $s$ is the spacing between these tracks and each turn being formed by four consecutive straight segments.

In practice, the manufactured BPSC still requires underpass tracks with the function of interconnecting the center of this planar coil to external terminals in order to connect it to an impedance analyzer for testing. Although BPSC generally has only one underpass track for each monofilar winding, the BPSC of Fig. 3 was designed with two underpass tracks for each monofilar winding in order to facilitate measurements between its terminals. The width $w_{o v}$ of the underpass track was chosen smaller than the track width $w$ of each monofilar PSC in order to minimize the parasitic capacitance that arises between these tracks. 


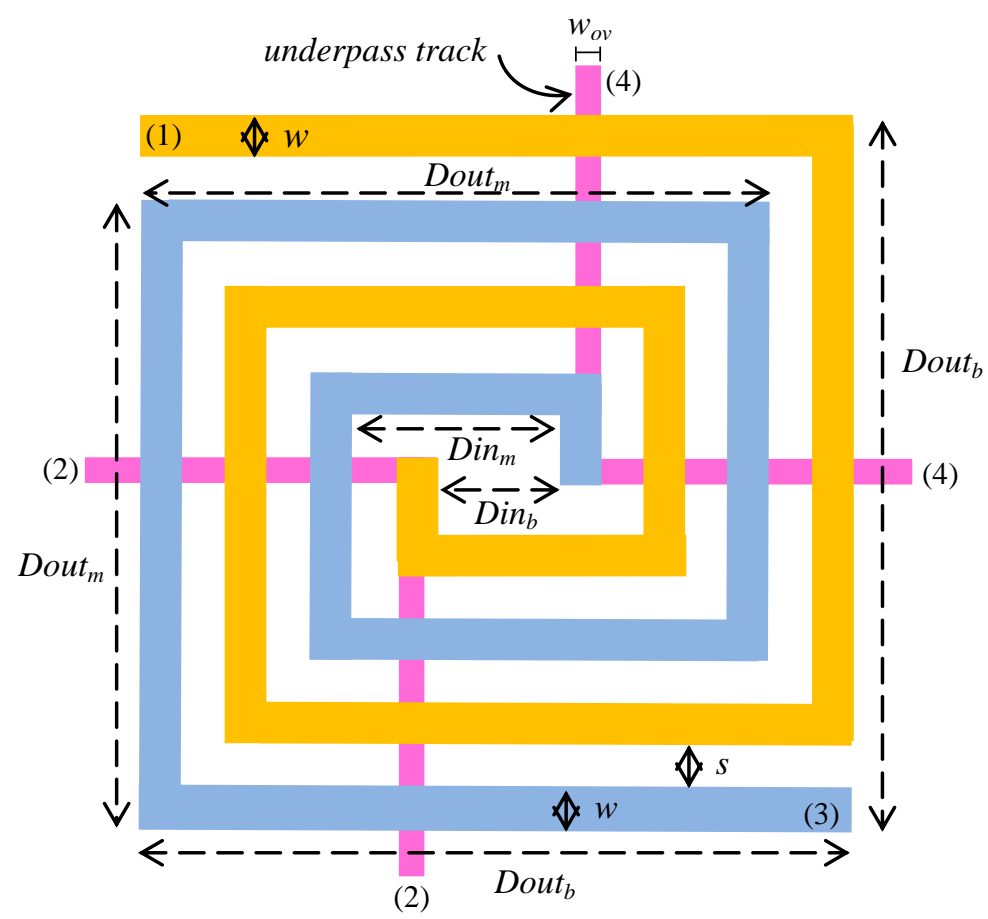

Fig. 3. Open square BPSC with $N=4$ turns, external side Dout $_{b}$, internal side $\operatorname{Din}_{b}$, formed by the monofilar PSC B1 with terminals (1)-(2) and by monofilar PSC B2 with terminals (3)-(4), each one with $N_{m}=2$ turns, track width $w$, external side Dout $_{m}$, internal side Din $_{m}$, spacing $s$ between tracks and with two underpass tracks of width $w_{o v}$.

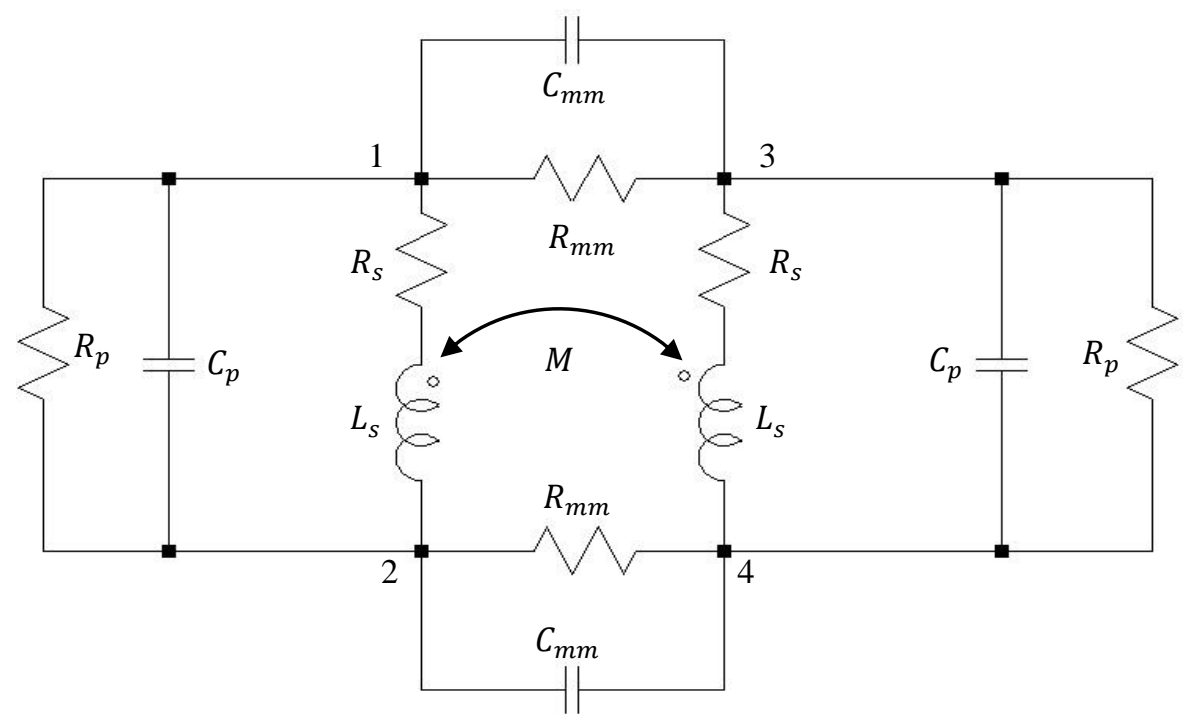

Fig. 4. Electrical model proposed for an open square BPSC.

In Fig. 4, an electrical model of an open square BPSC of symmetrical layout is proposed. All parameters of resistances, inductances and capacitances ( R, L, and C) distributed in B1 and B2 monofilar windings are considered identical, being

$R_{\mathrm{S}}$ series resistance of the tracks of each monofilar winding (B1 and $\mathrm{B} 2$ ),

$C_{p}$ total stray capacitance that arises between the turns of each monofilar winding,

$R_{p}$ resistance related to losses in dielectric materials and in the medium surrounding BPSC's tracks 
and that arises between turns of each monofilar winding,

$C_{m}$ mutual capacitance that arises between B1 and B2 monofilar windings and

$R_{m}$ resistance related to losses in dielectric materials and in the medium surrounding BPSC's tracks and that arises between $\mathrm{B} 1$ and $\mathrm{B} 2$ monofilar windings.

Resistance $R_{m}$ and capacitance $C_{m}$ are distributed in the electrical model of Fig. 4 into two parts and are referred as $R_{m m}$ and $C_{m m}$, being

$$
C_{m m}=0.5 C_{m}
$$

and

$$
R_{m m}=2 R_{m}
$$

Although resistances $R_{s}, R_{m m}$ and $R_{p}$ have a fundamental role in the impedance frequency response curve of the proposed model, in order to estimate the first valley $\omega_{1 \mathrm{v}}$ ( or $f_{1 \mathrm{v}}$ ) and the first resonance peak $\omega_{1 \mathrm{p}}\left(\right.$ or $\left.f_{1 \mathrm{p}}\right)$ for the open square BPSC, the model described in Fig. 4 will be simplified by excluding the resistive losses, since their effect on resonant frequencies can be considered negligible. This simplification will result in the impedance seen by terminals 1-4 as

$$
Z_{14}=\frac{j\left[-1+\omega^{2}\left(C_{p}+C_{m m}\right)\left(L_{s}+M\right)\right]}{2 \omega C_{m m}\left[1-\omega^{2} C_{p}\left(L_{s}+M\right)\right]},
$$

being $\omega$ the angular frequency and the respective resonant frequencies are

$$
\omega_{1 v}=\sqrt{\frac{1}{\left(C_{p}+C_{m m}\right)\left(L_{s}+M\right)}}=2 \pi f_{1 v}
$$

and

$$
\omega_{1 p}=\sqrt{\frac{1}{C_{p}\left(L_{s}+M\right)}}=2 \pi f_{1 p}
$$

As an example, Fig. 5 shows the $Z_{14}$ impedance modulus and phase curves obtained by electromagnetic simulations and measurements made by an impedance analyzer, for an open square BPSC with $N=28$, manufactured in double-sided PCB, with FR-4 substrate, coated with solder mask, $w=0.55 \mathrm{~mm}, w_{o v}=0.25 \mathrm{~mm}, s=0.45 \mathrm{~mm}$, Dout $_{b}=65.55 \mathrm{~mm}$ and $\operatorname{Din}_{b}=10.45 \mathrm{~mm}$. As will be shown in section III, the present study of BPSC electrical parameters can estimate the first valley and the first resonance peak with an error of less than $5 \%$. 
Journal of Microwaves, Optoelectronics and Electromagnetic Applications, Vol. 17, No. 3, September 2018324 DOI: http://dx.doi.org/10.1590/2179-10742018v17i31254



Fig. 5. Module and phase curves of $Z_{14}$ versus frequency $f$ to simulated and measured data of an open square BPSC with $N=28$ and localization of the first valley $\left(f_{1 v}\right)$ and of the first resonance peak $\left(f_{1 p}\right)$.

\section{A. Inductances and Magnetic Coupling Factor}

For an open square BPSC of symmetrical layout which inductances of the B1 and B2 monofilar windings are considered identical, self-inductance $L_{s}$ can be determined by equation

$$
L_{s}=0.635 \mu_{0} N_{m}^{2} d_{\text {avg }}\left[\ln \left(\frac{2.07}{t_{p m}}\right)+0.18 t_{p m}+0.13 t_{p m}^{2}\right]
$$

as presented in [13] for square PSCs and the mutual inductance $M$ by the equation

$$
M=\frac{L_{b}-2 L_{s}}{2}
$$

being

$$
\begin{gathered}
L_{b}=0.635 \mu_{0} N^{2} d_{\text {avg }}\left[\ln \left(\frac{2.07}{t_{p b}}\right)+0.18 t_{p b}+0.13 t_{p b}^{2}\right] \\
d_{\text {avg }}=0.5\left(\text { Dout }_{m}+\text { Din }_{m}\right)=0.5\left(\text { Dout }_{b}+\text { Din }_{b}\right) \\
t_{p m}=\left(\text { Dout }_{m}-\text { Din }_{m}\right) /\left(\text { Dout }_{m}+\text { Din }_{m}\right) \\
t_{p b}=\left(\text { Dout }_{b}-\text { Din }_{b}\right) /\left(\text { Dout }_{b}+\text { Din }_{b}\right) \\
\text { Dout }_{m}=\text { Dout }_{b}-(s+w)
\end{gathered}
$$

and

$$
\operatorname{Din}_{m}=\operatorname{Din}_{b}+(s+w),
$$

where $\mu_{0}=4 \pi 10^{-7} \mathrm{H} / \mathrm{m}$ is the air magnetic permeability, $d_{\text {avg }}$ is the BPSC's average side, $L_{b}$ is its total inductance between terminals 1-4 with terminals 2-3 interconnected, $t_{p m}$ is the fill ratio of each monofilar PSC and $t_{p b}$ is the BPSC's fill ratio.

BPSC is analyzed in the closed-circuit configuration only for the calculation of total inductance $L_{b}$. All other inductances and capacitances of the proposed model will be determined with the BPSC in open-circuit configuration. 
Again, considering the self-inductances of the monofilar windings B1 and B2 as being identical and using equations (6) and (7), the magnetic coupling factor is determined by the equation

$$
k=\frac{M}{L_{s}} .
$$

The inductance calculation presented in [13] is based on approximating the sides of each monofilar PSC as current sheets, being its maximum error limited to $8 \%$ for PSCs with $s / w<3$.

\section{B. Capacitances}

Mutual capacitance $C_{m}$ is a capacitance that arises between pairs of tracks belonging to B1 and B2 monofilar PSCs which are mutually coupled.

This capacitance will be determined considering that the BPSC can be formed by CPW lines with finite-width lateral ground plane, assuming such ground plane width equal to $w$ [14]-[15].

It considers, initially, an alternating signal source applied to terminals 1-3 of Fig. 3 with potential $V_{1}$ being instantaneously greater than $V_{3}$. For the calculation of capacitance $C_{m}$ only the contributions of pairs of capacitances $C_{t}$ that form between adjacent parallel tracks and that can represent a threewire line $(\mathrm{CPW})$ are taken into account. From this premise, capacitance pairs $C_{t}$ are distributed along almost all tracks, with the exception of the first two outermost tracks and the last two innermost ones of the monofilar PSC B1, according to Fig. 6. Thus, the average length $l_{a v}$ used for the calculation of $C_{m}$ is

$$
l_{a v}=4 \text { Dout }_{b}\left(N_{m}-1\right)-4 N_{m}\left(2 N_{m}-3\right)(s+w)-4\left(N_{m} w+s\right) .
$$

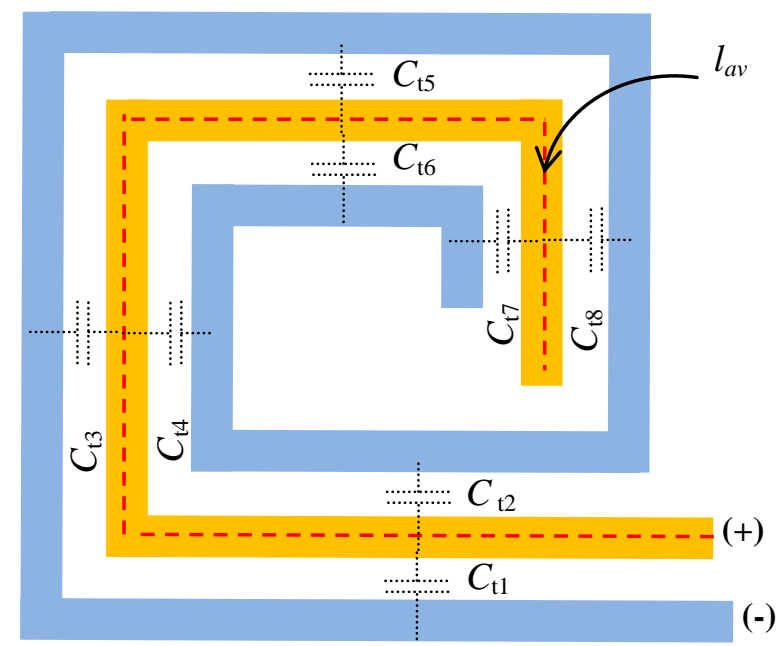

Fig. 6. Distributed capacitances $C_{t}$ along of three-wire parallel tracks that totalize an average length $l_{a v}$. These three-wire parallel tracks form four CPW lines, obtained from the BPSC with $N=4$ of Fig. 3, discounting the first two outermost tracks and the last two innermost ones of monofilar PSC B1.

Fig. 7 shows the cross-section of only three adjacent parallel tracks of a BPSC which represent a CPW line with finite-width lateral ground plane surrounded by three dielectric materials: the top and bottom layers contain a solder mask with dielectric constant, respectively, $\varepsilon_{\mathrm{r} 1}$ and $\varepsilon_{\mathrm{r} 3}$ and between 
these two layers there is an FR-4 substrate with dielectric constant $\varepsilon_{\mathrm{r} 2}$. It should also be considered that these dielectric materials have relative heights $t_{1}, t_{2}$ and $t_{3}$ and that the medium surrounding the BPSC is the air.

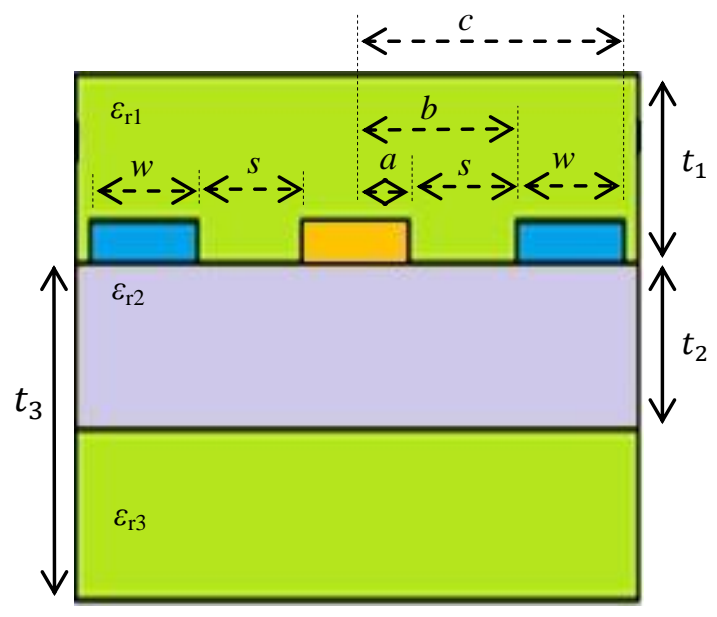

Fig. 7. Cross section of a CPW for modeling of mutual capacitance $C_{m}$ of a BPSC.

Applying the conformal mapping and superposition of partial capacitances techniques to the scheme shown in Fig. 7, the capacitance per unit length of a CPW can be expressed as [15]

$$
C_{C P W}=\varepsilon_{\text {ref }} C_{o},
$$

where $\varepsilon_{r e f}$ is the effective relative permittivity and $C_{o}$ is the partial capacitance of the CPW in free space (vacuum or air).

$$
C_{o}=4 \frac{\varepsilon_{o} K\left(k_{o}^{\prime}\right)}{K\left(k_{o}\right)}
$$

where $\varepsilon_{o}$ is the electric permittivity of the vacuum $\left(8.8542 .10^{-12} \mathrm{~F} / \mathrm{m}\right)$ and $K\left(k_{\mathrm{o}}\right)$ and $K\left(k_{\mathrm{o}}^{\prime}\right)$ are the complete elliptic integrals of the first kind that can be calculated by equations

$$
\begin{gathered}
k_{o}=\frac{c}{b} \sqrt{\frac{d}{e},} \\
k_{o}^{\prime}=\sqrt{1-k_{o}^{2},} \\
d=b^{2}-a^{2}, \\
e=c^{2}-a^{2}, \\
c=1.5 w+s, \\
b=s+a,
\end{gathered}
$$

and

$$
a=0.5 w \text {. }
$$

The effective relative permittivity $\varepsilon_{r e f}$ is determined by equation

$$
\varepsilon_{r e f}=1+\left(\varepsilon_{r 1}-1\right) q_{1}+\left(\varepsilon_{r 2}-\varepsilon_{r 3}\right) q_{2}+\left(\varepsilon_{r 3}-1\right) q_{3},
$$


being

$$
\begin{gathered}
q_{1}=\frac{K\left(k_{o}\right) K\left(k_{11}^{\prime}\right)}{2 K\left(k_{o}^{\prime}\right) K\left(k_{11}\right)}, \\
q_{2}=\frac{K\left(k_{o}\right) K\left(k_{22}^{\prime}\right)}{2 K\left(k_{o}^{\prime}\right) K\left(k_{22}\right)}, \\
q_{3}=\frac{K\left(k_{o}\right) K\left(k_{33}^{\prime}\right)}{2 K\left(k_{o}^{\prime}\right) K\left(k_{33}\right)}, \\
k_{i i}=\frac{\sinh \left(\frac{\pi c}{2 t_{i}}\right)}{\sinh \left(\frac{\pi b}{2 t_{i}}\right)} \sqrt{\frac{\sinh ^{2}\left(\frac{\pi b}{2 t_{i}}\right)-\sinh ^{2}\left(\frac{\pi a}{2 t_{i}}\right)}{\sinh ^{2}\left(\frac{\pi c}{2 t_{i}}\right)-\sinh ^{2}\left(\frac{\pi a}{2 t_{i}}\right)}},
\end{gathered}
$$

and

$$
k_{i i}^{\prime}=\sqrt{1-k_{i i}^{2}}
$$

where $q_{i}$ is the filling factor, $k_{i i}$ and $k_{i i}^{\prime}$ are elliptic integral moduli and $t_{i}$ is the relative height of the dielectric layer $i$, being $i$ varying from 1 to 3 is the indice associated to each one of the three dielectric layers shown in Fig. 7.

Thus, multiplying the equation (16) by (15), the mutual capacitance of the BPSC can be determined from the equation

$$
C_{m}=C_{C P W} l_{a v}
$$

The calculation of the mutual capacitance of BPSCs using CPW lines approach, proposed in this paper, will be compared with measured values only in section III. However, in order to test the validity of equation (31), comparisons were made with results obtained by EM simulations and with the calculation of mutual capacitances modeled by coplanar striplines (CPS) used in [8].

For this purpose, EM simulations were performed with three groups of 10 BPCSs, according to Tables I to III, in order to determine the mutual capacitance values $C_{m E M}$. For each group of 10 BPSCs, $w, s, \operatorname{Din}_{b}$ and the parameters of Table IV were kept fixed, the only variables being $N_{m}$ and Dout $_{b}$, according to Tables I to III. The fixed parameters described in Table IV, common to all three groups of BPSCs are associated with Fig. 7, being $t$ the metal track thickness of the BPSC and $\tan D_{i}$ ( $i=1$ to 3 ) the loss tangent of each dielectric layer $i$ of the BPSC.

TABLE I. GROUP 1 - BPSCs

\begin{tabular}{cl}
\hline $\boldsymbol{N}_{\boldsymbol{m}}$ & 5 to 14 \\
\hline $\boldsymbol{w}$ & $0.80 \mathrm{~mm}$ \\
\hline $\boldsymbol{s}$ & $0.20 \mathrm{~mm}$ \\
\hline Dout $_{\boldsymbol{b}}$ & $29.80 \mathrm{~mm}$ to $65.8 \mathrm{~mm}$ \\
\hline Din $_{\boldsymbol{b}}$ & $10.2 \mathrm{~mm}$ \\
\hline
\end{tabular}

TABLE II. GROUP 2 - BPSCs

\begin{tabular}{cl}
\hline$N_{\boldsymbol{m}}$ & 5 to 14 \\
\hline $\boldsymbol{w}$ & $0.55 \mathrm{~mm}$ \\
\hline $\boldsymbol{s}$ & $0.45 \mathrm{~mm}$ \\
\hline Dout $_{\boldsymbol{b}}$ & $29.55 \mathrm{~mm}$ to $65.55 \mathrm{~mm}$ \\
\hline Din $_{\boldsymbol{b}}$ & $10.45 \mathrm{~mm}$ \\
\hline
\end{tabular}

TABLE III. GROUP 3 - BPSCs

\begin{tabular}{cl}
\hline $\boldsymbol{N}_{\boldsymbol{m}}$ & 5 to 14 \\
\hline $\boldsymbol{w}$ & $0.55 \mathrm{~mm}$ \\
\hline $\boldsymbol{s}$ & $0.20 \mathrm{~mm}$ \\
\hline Dout $_{\boldsymbol{b}}$ & $34.30 \mathrm{~mm}$ to $61.30 \mathrm{~mm}$ \\
\hline Din $_{\boldsymbol{b}}$ & $19.70 \mathrm{~mm}$ \\
\hline
\end{tabular}


Journal of Microwaves, Optoelectronics and Electromagnetic Applications, Vol. 17, No. 3, September 2018328 DOI: http://dx.doi.org/10.1590/2179-10742018v17i31254

TABLE IV. ALL GROUPS - FIXED PARAMETERS

\begin{tabular}{cl}
\hline $\boldsymbol{t}$ & $39 \mu \mathrm{m}$ (metal track thickness) \\
\hline $\boldsymbol{t}_{\mathbf{1}}$ & $50 \mu \mathrm{m}$ (top solder mask) \\
\hline $\boldsymbol{t}_{\boldsymbol{2}}$ & $1.58 \mathrm{~mm}$ (FR-4 substrate) \\
\hline $\boldsymbol{t}_{\mathbf{3}}$ & $1.62 \mathrm{~mm}$ (bottom solder mask of $40 \mu \mathrm{m})$ \\
\hline $\boldsymbol{\varepsilon}_{\boldsymbol{r} \mathbf{1}}$ & 4.00 \\
\hline $\boldsymbol{\varepsilon}_{\boldsymbol{r} \mathbf{2}}$ & 4.85 \\
\hline $\boldsymbol{\varepsilon}_{\boldsymbol{r} \mathbf{3}}$ & 4.00 \\
\hline $\tan \boldsymbol{D}_{\mathbf{1}}$ & 0.035 \\
\hline $\tan \boldsymbol{D}_{\mathbf{2}}$ & 0.018 \\
\hline $\tan \boldsymbol{D}_{\mathbf{3}}$ & 0.035 \\
\hline
\end{tabular}

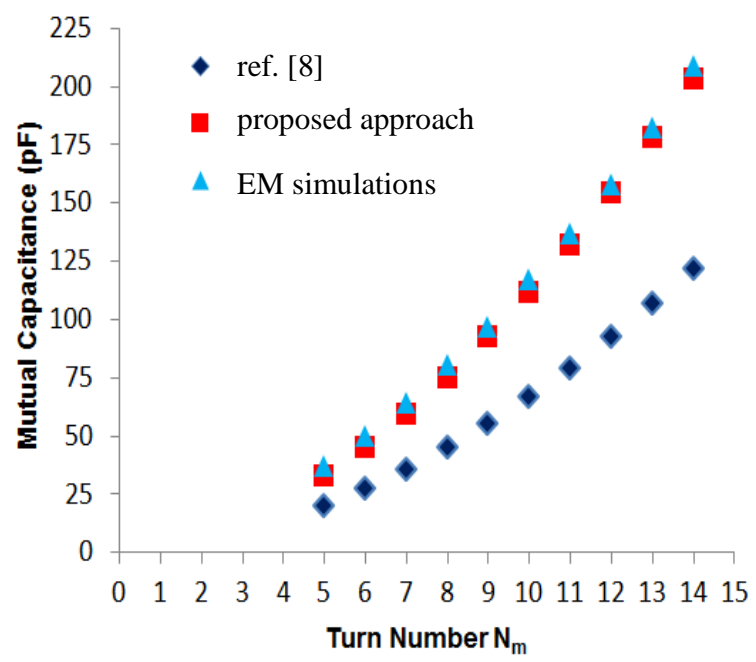

(a)

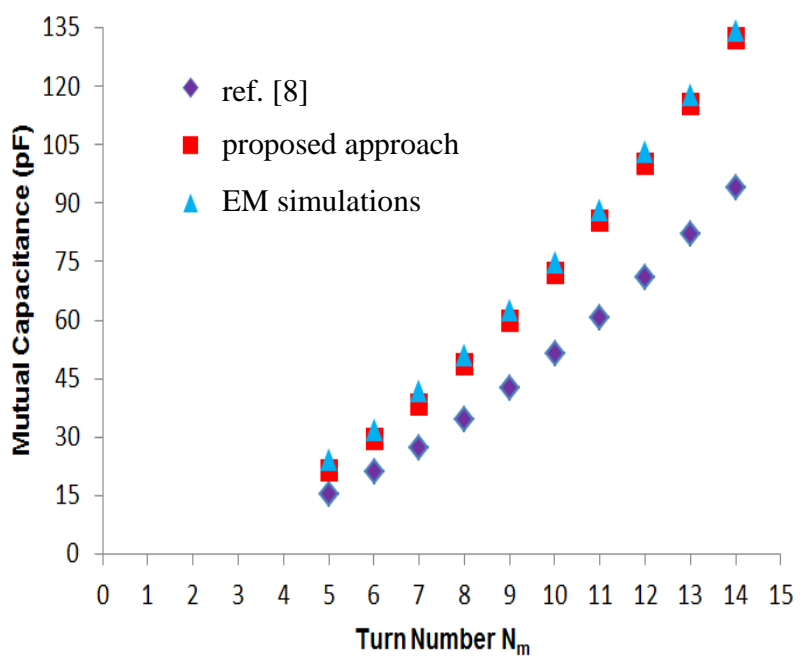

(b)



(c)

Fig. 8. Plots of mutual capacitance versus $N_{m}$ for BPSCs (a) from group 1, (b) from group 2 and (c) from group 3: for $C_{m}$ using the CPW lines approach proposed in this paper, for $C_{m E M}$ using EM simulations and by CPS lines used in [8]. 
Next, the values of $C_{m}$ obtained by CPW lines approach and those obtained by EM simulation, for the three groups of BPSCs described in Tables I to III, were compared with the respective mutual capacitances modeled by CPS lines used in [8]. Results are presented in Fig. 8.

As shown in Fig. 8, the modeling of mutual capacitances using CPW lines proposed in this paper results in values of $C_{m}$ that are very close to the results obtained by EM simulations. For BPSCs of groups 1 to 3 , the error of $C_{m}$ relative to $C_{m E M}$ ranged from $1.69 \%$ to $12.92 \%$, whereas the error using the CPS lines approach proposed by [8] ranged from $30.20 \%$ to $46.93 \%$.

According to equation (4), $C_{m}$ is related only with the first resonance valley of the open BPSC, whereas the total stray capacitance $C_{p}$ is associated with the first valley and with the first resonance peak, as presented in equation (5), and is defined as

$$
C_{p}=C_{s}+C_{o v}
$$

where $C_{s}$ is the stray capacitance, named also as self-capacitance [4], that arises between the turns of each monofilar spiral winding present in the BPSC's top layer, and $C_{o v}$ is the stray capacitance that arises between the top layer tracks and the underpass tracks, according to Fig.9.

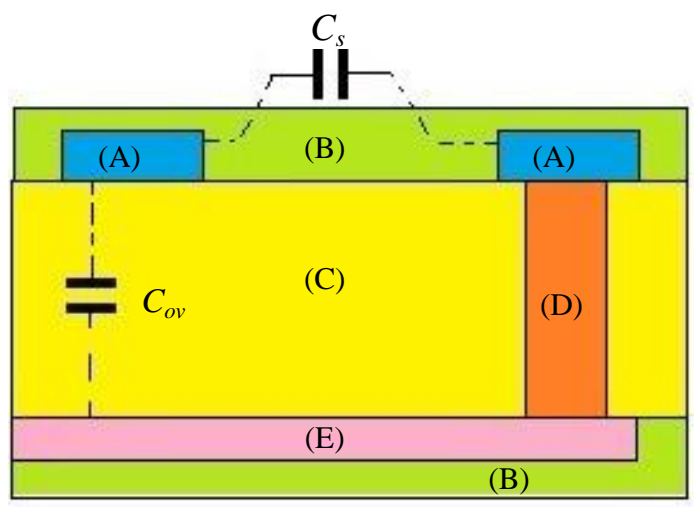

Fig. 9. Cross section of a BPSC showing where the capacitances $C_{s}$ and $C_{o v}$ arise in the monofilar PSC B2: (A) are tracks of the PSC B2, (B) solder mask layers, (C) substrate layer and (D) a via which interconnects the center of the flat coil to the underpass track (E). For simplicity, in this figure one single underpass track was represented.

While mutual capacitance $C_{m}$ was calculated using a distance $s$ between adjacent tracks, the selfcapacitance $C_{s}$ is associated with a distance $2 s$ between adjacent tracks and not $2 s+w$. As shown in Fig. 6, between two spacing $s$ there is a conductive track of width $w$ that does not contribute to the calculation of self-capacitance, because the electric field inside the conductor is null. Thus, as a first estimate, the self-capacitance of monofilar PSC could, in principle, be calculated as

$$
C_{s m}=0.5 C_{m}=C_{m m} .
$$

However, this first approximation for self-capacitance is not yet consistent with results obtained by EM simulation, because for the calculation of $C_{m}$ it was assumed a constant potential difference (p.d.) in amplitude between monofilar PSCs B1 and B2 along the full length of such PSCs. On the other hand, applying a voltage source, for example, only to the terminals of the monofilar PSC B2, the p.d. 
which arises between its pairs of adjacent metal tracks will not be constant along the whole length of the planar spiral winding, but will gradually decrease, when comparing the outermost with the innermost turns of the PSC. Thus, the parasitic capacitances $C_{t s}$ distributed along the monofilar PSC B2 shown in Fig. 10 depend not only on geometric parameters and dielectric media as predicted in equation (31), but also depend on p.d. which is established between each pair of tracks or even of the respective portions of energy stored by the electric field between these metal tracks [16].

This gradual voltage drop along the spiral winding makes the $C_{s}$ self-capacitance value of each monofilar PSC to be significantly smaller than the first estimate described in equation (33) mainly for PSCs with high number of turns. However, it is possible to take advantage of equation (33) by multiplying it by a degeneration factor $\alpha$ obtained from EM simulations and with the aid of statistical data processing software [17]. This is the strategy adopted in this paper for the determination of $C_{s}$.

Thus,

$$
C_{s}=\alpha C_{m m}
$$

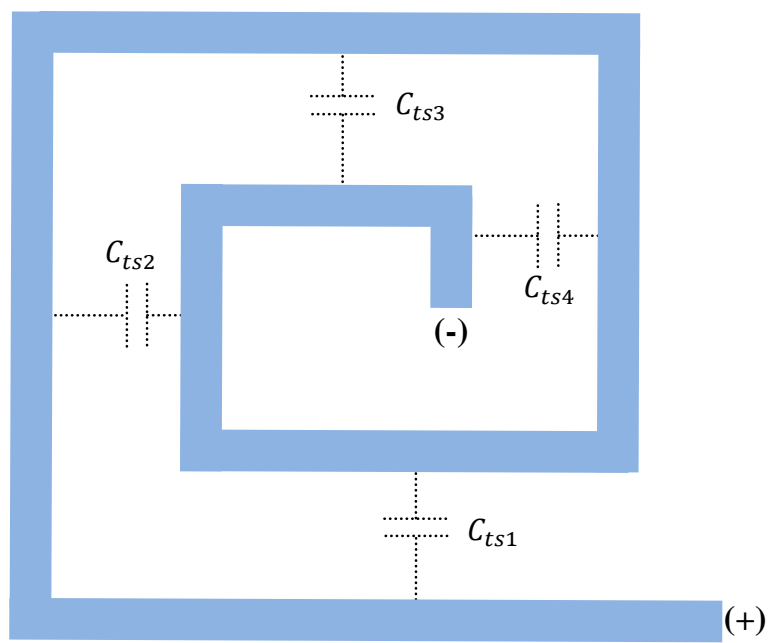

Fig. 10. Distribution of stray capacitances $C_{t s}$ along adjacent parallel tracks, after connecting a voltage source between the terminals of the monofilar PSC B2 with $N_{m}=2$.

In order to determine degeneration factors, EM simulations will be performed again with the three groups of 10 BPCSs described in Tables I to III. However, this time, each BPSC will be simulated without underpass tracks, aiming to determine self-capacitances $C_{S E M}$ and mutual capacitances $C_{m E M}$.

For each BPSC associated to groups 1 to 3 , a degeneration factor was determined as a function of $N_{m}$ and defined as,

$$
\alpha_{E M}=\frac{C_{S E M}}{0.5 C_{m E M}} .
$$

The ten $\alpha_{E M}$ and $N_{m}$ values for each group of BPSCs were introduced into the LAB FIT software which provided a fitting equation $\alpha$ and its coefficients for the study of the data under analysis [18].

Thus, 
Journal of Microwaves, Optoelectronics and Electromagnetic Applications, Vol. 17, No. 3, September 2018331 DOI: http://dx.doi.org/10.1590/2179-10742018v17i31254

$$
\alpha=\frac{k_{B} N_{m}+k_{A}}{N_{m}^{2}}
$$

where constants $k_{A}$ and $k_{B}$ are represented in Table V.

TABLE V. COEFICIENTS $k_{A}$ AND $k_{B}$ OF THE DEGENERATION FACTOR $\alpha$

\begin{tabular}{ccc}
\hline GROUP & $\boldsymbol{k}_{\boldsymbol{A}}$ & $\boldsymbol{k}_{\boldsymbol{B}}$ \\
\hline 1 & 0.1106 & 0.2275 \\
\hline 2 & 0.1671 & 0.2652 \\
\hline 3 & 0.1070 & 0.3415 \\
\hline
\end{tabular}

The degeneration factors versus $N_{m}$ for groups 1 to 3 are shown in Figs. 11 to 13 . These factors are subsequently substituted in equation (34) in order to determine the estimated $C_{s}$ of each BPSC. The interval $14 \geq N_{m} \geq 5$ was chosen, for the three groups, so that the error in $C_{s}$ was limited to $13 \%$ regarding the respective capacitance values $C_{S E M}$ obtained by EM simulation.

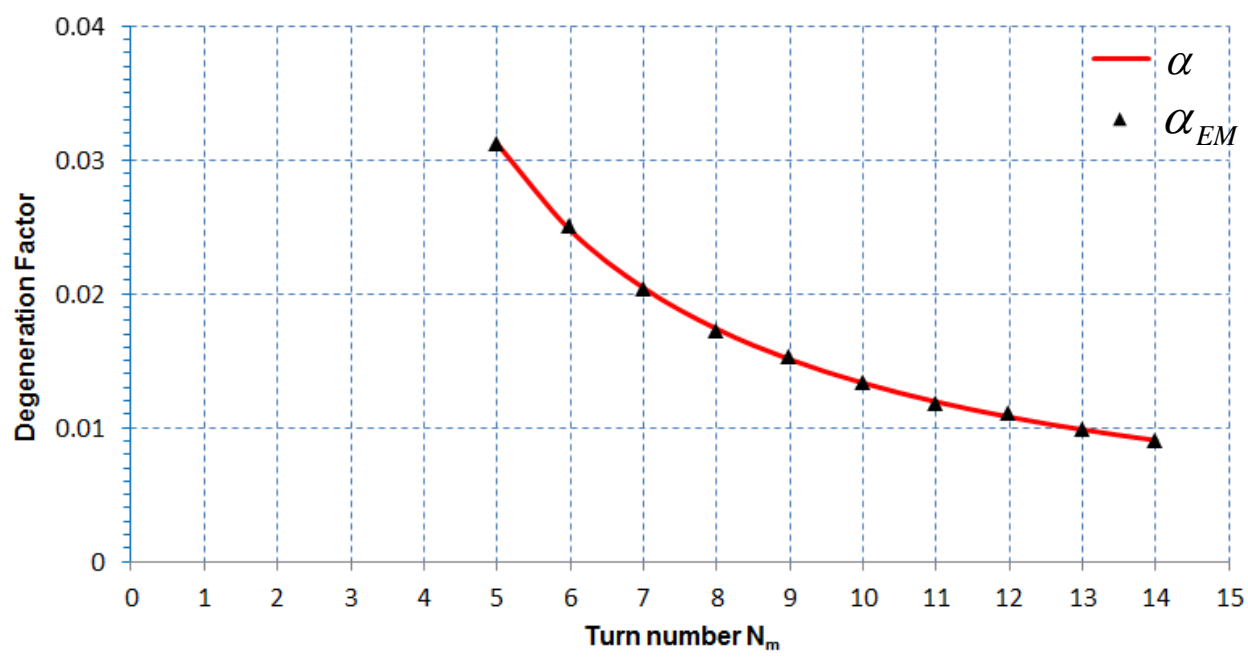

Fig. 11. Degeneration factor versus $N_{m}$ curve for group 1 BPSCs.

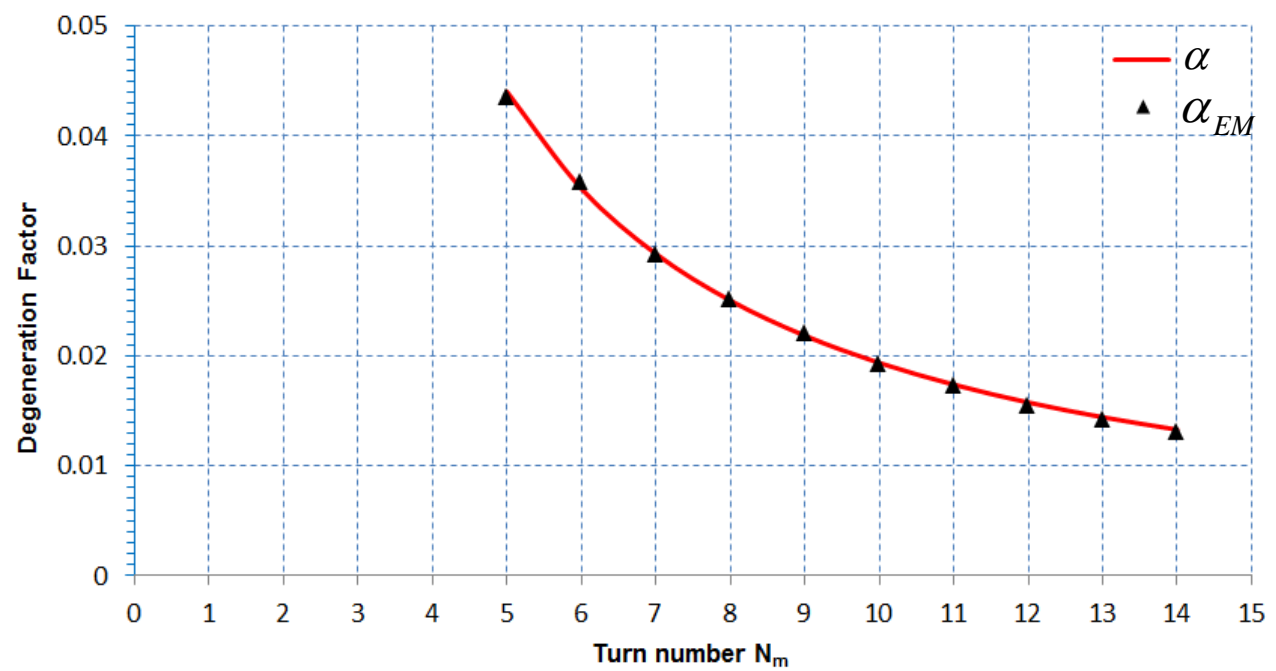

Fig. 12. Degeneration factor versus $N_{m}$ curve for group 2 BPSCs. 


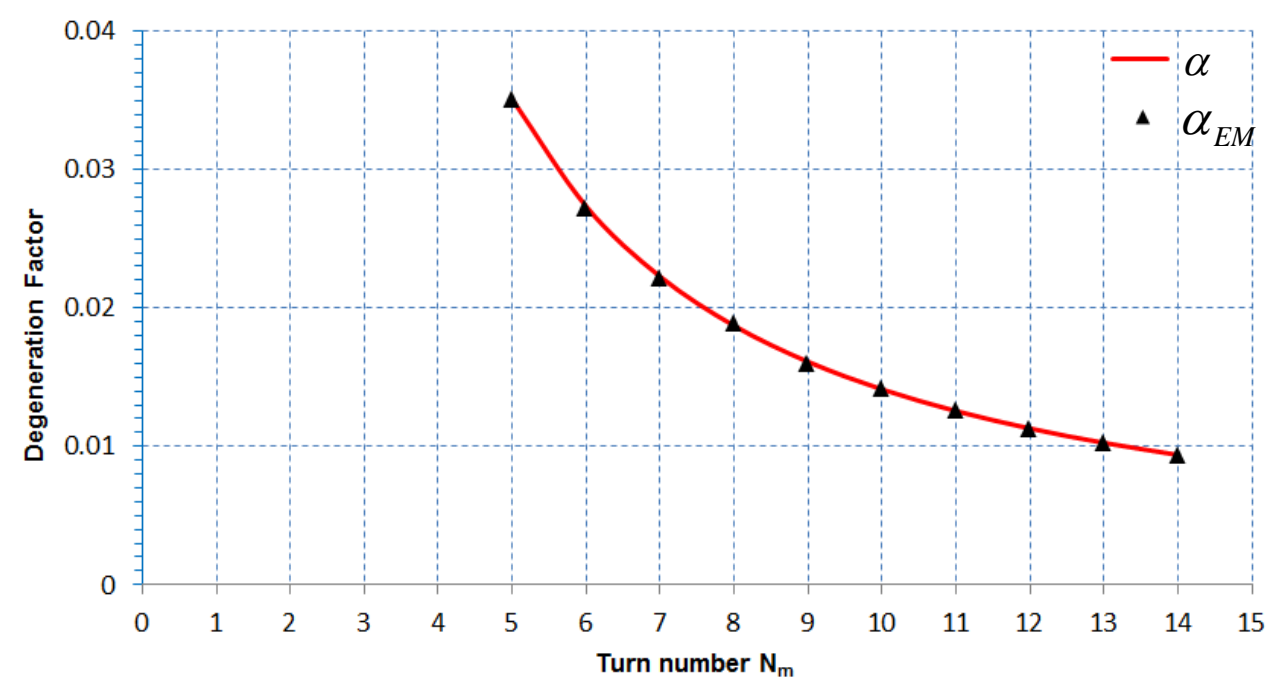

Fig. 13. Degeneration factor versus $N_{m}$ curve for group 3 BPSCs.

The curves shown in Figs. 11 to 13 are useful in the way of determining capacitances $C_{s}$ more quickly, without the necessity to perform new EM simulations, as long as the BPSC to be manufactured has parameters within the limits described in Tables I to IV. Therefore, a set of BPSCs with parameters outside the limits which have been mentioned in these tables will result in different coefficients $k_{A}$ and $k_{B}$ from those shown in Table V.

Results obtained for self-capacitances $C_{s}$ using the methodology adopted in this paper were compared with the respective $C_{S E M}$ obtained by EM simulations and were also compared with selfcapacitances obtained by the CPS lines approach used in [8] and [19]. These results are presented in Fig. 14, where a good agreement of $C_{s}$ with the simulated results can be observed. For BPSCs from groups 1 to 3, the error of $C_{s}$ regarding $C_{S E M}$ ranged between $0.044 \%$ and $13.070 \%$. On the other hand, the calculation of self-capacitances by CPS lines presented in [8] generated values between 22 and 110 times higher than $C_{S E M}$, because in [8] the voltage drop along the spiral winding as well as the degeneration factors were not taken into account. Thus, comparing results described in Fig. 14 and Fig. 8, the study presented in [8] is more suitable for the determination of mutual capacitances than for self-capacitances, although the error was still higher than $30 \%$ for mutual capacitances. In [19], which also used the approach of CPS lines, it was already considered the voltage drop per turn, but an arbitrary degeneration factor equal to $\left(N_{m}\right)^{-1}$ was adopted resulting in self-capacitances between 2.8 and 8 times greater than $C_{S E M}$. 


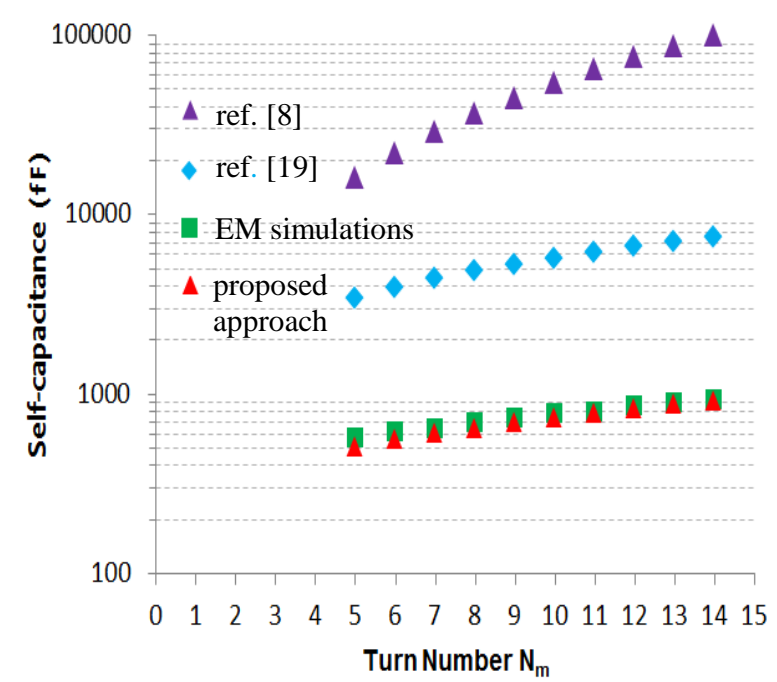

(a)

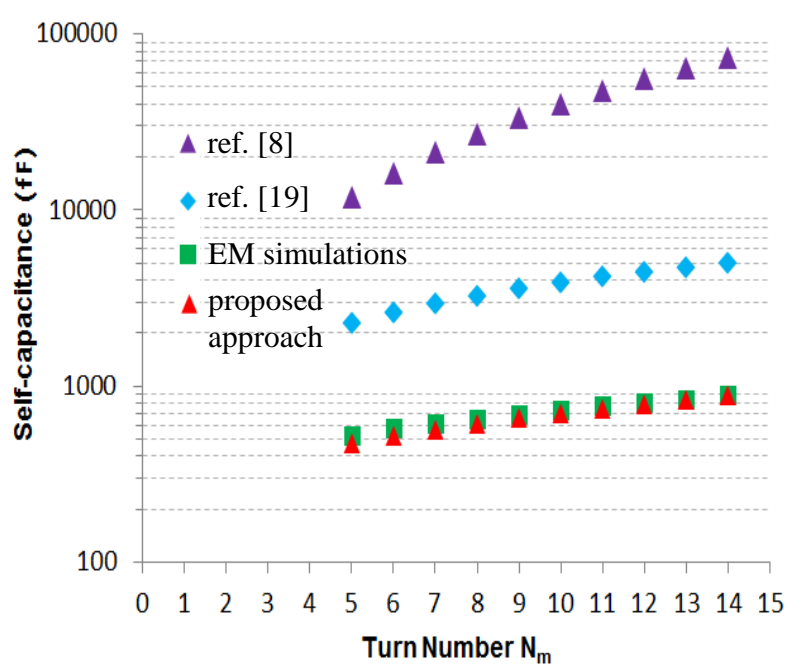

(b)

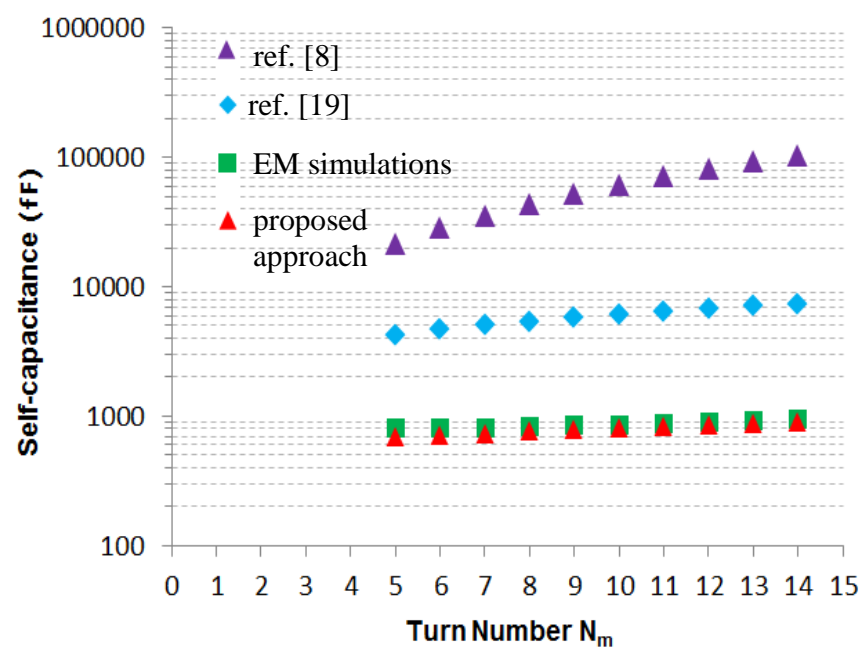

(c)

Fig. 14. Plots of self-capacitance versus $N_{m}$ for BPSCs (a) from group 1, (b) from group 2 and (c) from group 3: for $C_{s}$ using CPW lines and degeneration factor approaches adopted in this paper, for $C_{S E M}$ using EM simulations and by modeling of CPS lines used in [8] and [19].

So far all the inductance and capacitance analysis described in this section has neglected the influence of the underpass tracks. However, in order to determine the total parasitic capacitance $C_{p}$, it is necessary to estimate the stray capacitance $C_{o v}$.

The capacitance $C_{o v}$, shown in Fig. 9, which arises between tracks of the BPSC's top layer and underpass tracks, can be estimated by equation

$$
C_{o v}=k_{o v} \varepsilon_{o} \varepsilon_{r 2} \frac{A_{o v}}{t_{2}}
$$

where

$$
A_{o v}=N_{m} w w_{o v}
$$

is the total area of all pairs of tracks overlapping between the top layer and underpasses, being the width $w_{o v}$ of the underpass track fixed at $0.25 \mathrm{~mm}$ for all BPSCs studied in this paper in order to minimize the impact of $C_{o v}$ about the total stray capacitance $C_{p}$. The effect of fringing fields is taken 
into account by the fitting factor $k_{o v}$ obtained by electromagnetic simulations which value, for each group of BPSCs, is described in Table VI.

TABLE VI. COEFICIENT
\begin{tabular}{cc}
\hline GROUP & $\boldsymbol{k}_{\boldsymbol{o v}}$ \\
\hline 1 & 8.83 \\
\hline 2 & 10.95 \\
\hline 3 & 10.27 \\
\hline
\end{tabular}

Equation (37) is valid for the groups of BPSCs described in Tables I to III with a maximum error of $10 \%$ regarding the $C_{o v}$ obtained by EM simulation.

After determining $C_{o v}$ by means of the equation (37) and $C_{s}$ using equation (34), the total parasitic capacitance $C_{p}$ of each monofilar winding of the BPSC is then determined by equation (32).

\section{METHODOLOGY, RESULTS AND DISCUSSIONS}

\section{A. Methodology}

In order to validate the lossless model and the theory presented in the previous section, three double-sided BPSCs on FR-4 substrate, coated with solder mask were manufactured. The common specifications of these BPSCs are defined in Table IV and the individual specifications of each one are set in Table VII.

TABLE VII. MANUFACTURED BPSCS

\begin{tabular}{cccccc}
\hline $\boldsymbol{N}$ & BIFILAR & $\boldsymbol{w}(\mathbf{m m})$ & $\boldsymbol{s}(\mathbf{m m})$ & Dout $_{\boldsymbol{b}}(\mathbf{m m})$ & $\boldsymbol{D i n}_{\boldsymbol{b}}(\mathbf{m m})$ \\
\hline 20 & BPSC-1 & 0.80 & 0.20 & 49.80 & 10.20 \\
24 & BPSC-2 & 0.55 & 0.20 & 55.30 & 19.70 \\
28 & BPSC-3 & 0.55 & 0.45 & 65.55 & 10.45 \\
\hline
\end{tabular}

BPSCs with 20, 24 and 28 turns were manufactured, so that the first resonances (peak and valley) may be a frequency spectrum measurable by the Keysight (Agilent) 4294A precision impedance analyzer.

Tables were made with the main electrical parameters of the BPSC in order to establish a comparative analysis between the results obtained by the proposed model, by EM simulation as well as for values measured by the 4294A impedance analyzer, for the three BPSCs described in Table VII.

The electrical parameters of the lossless model were obtained according to the theory presented in section II and by an algorithm implemented in MATLAB [20].

\section{1. $\quad$ Simulated Parameters}

Parameters $C_{p}, L_{s}$ and $M$ were obtained by EM simulations (Method of Moments - MoM) using Keysight's ADS software [21] that generates a $S$ parameter matrix which is later converted to a $Z$ 
impedance matrix or to a $Y$ admittance matrix. After determination of $L_{s}$ and $M$, the simulated magnetic coupling factor $k$ can be determined by applying equations (40) and (41) in equation (14).

The capacitance $C_{p}$ is obtained by the equation

$$
C_{p}=\frac{1}{\left(L_{s}+M\right) \omega_{1 p}^{2}}
$$

being $\omega_{1 p}$ the angular frequency where the first resonance peak occurs and $L_{s}$ and $M$ are determined by the equation

$$
L_{s}=\frac{\mathfrak{I}\left(Z_{11}\right)}{\omega}
$$

and

$$
M=\frac{\mathfrak{J}\left(Z_{12}\right)}{\omega},
$$

considering the BPSC as a quadripole (port 1 formed by terminals 1-2 and port 2 formed by terminals 3-4 of the BPSC) and $Z_{11}$ and $Z_{12}$ are elements of the impedance matrix $Z$ of the quadripole.

The simulated capacitance $C_{s}$ is also determined in the same way described above for $C_{p}$ using equation (39), but the simulation must be done without the underpass tracks and simulated $C_{o v}$, which in turn, is obtained by subtracting $C_{p}$ from $C_{s}$.

In order to obtain simulated $C_{m}$, the layout of each BPSC was drawn in the Keysight ADS with a short circuit between terminals 1-2 of the PSC B1 and also in the terminals 3-4 of the PSC B2 aiming to minimize the influence of the $R_{p}-C_{p}$ and $L_{s}-R_{s}$ branches on the simulated $C_{m}$. Next, a single port was connected between B1 and B2 windings of the BPSC, and later, the $S$ parameter matrix was converted into a $Y$ admittance matrix.

Thus,

$$
C_{m}=\frac{\mathfrak{I}(Y)}{\omega} .
$$

For comparative analysis with the electric parameters obtained for the model, $C_{p}$ and $C_{m}$ values obtained by EM simulation were determined at $1 \mathrm{MHz}$, because the manufacturer of the FR-4 substrate and the solder mask provide this test frequency for the dielectric constant as well as for the loss tangent. $L_{s}, M$ and $k$ were also obtained at $1 \mathrm{MHz}$ which is a region that provided stable values of inductances, since this frequency is relatively distant from the first resonance valley $f_{1 \mathrm{v}}$ of each BPSC analyzed.

\section{2. $\quad$ Measured Parameters}

$C_{m}, L_{s}$ and $M$ were measured according to the experimental procedure described by [22] for coreless planar transformers. In order to obtain measured $C_{m}$, a short circuit between terminals 1-2 of the PSC B1 and also in the terminals 3-4 of the PSC B2 are done for reasons already described in the previous subsection. Value measured of $L_{s}$ was obtained between terminals 1-2 and with terminals 3-4 in opencircuit. For determination of the mutual inductance $M$, the following procedure was adopted: opposite 
polarity terminals $2-3$ of the BPSC were initially short-circuited and the inductance $L_{14}$ seen by the terminals 1-4 was measured. Subsequently, the short circuit was removed between terminals 2-3. Next, terminals 2-4 of the same polarity were connected and the inductance $L_{13}$ seen by terminals 1-3 of the BPSC was measured [22].

From the measured values of inductances $L_{14}$ and $L_{13}$, the mutual inductance $M$ of the BPSC was determined using equation

$$
M=\frac{L_{14}-L_{13}}{4} .
$$

After the determination of $L_{s}, M$ and the measurement of the first resonance peak $f_{1 \mathrm{p}}$, the capacitance $C_{p}$ can be estimated using equation (5) and $k$, again, by equation (14).

The measurements of $C_{p}, C_{m}, L_{\mathrm{s}}, M$ and $k$ were also obtained at $1 \mathrm{MHz}$ for the same reason mentioned for the simulated parameters.

\section{B. Results and Discussions}

Tables VIII, IX and X show the main parameters $\left(C_{p}, C_{m}, L_{s}, M, k, f_{1 \mathrm{p}}\right.$ and $\left.f_{1 \mathrm{v}}\right)$ of BPSC-1, BPSC-2 and BPSC-3 obtained for the model, for the values measured by the impedance analyzer and by EM simulation. These tables also show, in the last two columns, the percentage difference or error of each modeled and simulated parameter regarding the measured values, where it is observed that the respective errors of all parameters of the proposed model are smaller than $10 \%$.

TABLE VIII. BPSC-1: COMPARATIVE ANALYSIS

\begin{tabular}{lccccc}
\hline & & & & \multicolumn{2}{c}{ Error (\%) } \\
Parameters & Model & Measurement & EM Simulation & Model & EM Simulation \\
\hline$C_{p}(\mathrm{pF})$ & 1.2209 & 1.3199 & 1.2658 & -7.5006 & -4.0988 \\
\hline$L_{s}(\mu \mathrm{H})$ & 3.2527 & 3.4090 & 3.2839 & -4.5849 & -3.6697 \\
\hline$M(\mu \mathrm{H})$ & 3.0598 & 2.9354 & 2.9620 & 4.2379 & 0.9062 \\
\hline$k$ & 0.9407 & 0.8611 & 0.9020 & 9.2440 & 4.7497 \\
\hline$C_{m}(\mathrm{pF})$ & 111.1200 & 113.9120 & 118.9686 & -2.4510 & 4.4390 \\
\hline$f_{1 \mathrm{p}}(\mathrm{MHz})$ & 57.3296 & 55.0002 & 56.6039 & 4.2353 & 2.9158 \\
\hline$f_{1 \mathrm{v}}(\mathrm{MHz})$ & 8.4066 & 8.2771 & 8.1705 & 1.5646 & -1.2879 \\
\hline
\end{tabular}

TABLE IX. BPSC-2: COMPARATIVE ANALYSIS

\begin{tabular}{lccccc}
\hline Parameters & Model & Measurement & EM Simulation & Model & EM Simulation \\
\hline$C_{p}(\mathrm{pF})$ & 1.3200 & 1.4327 & 1.3640 & -7.8662 & -4.7951 \\
\hline$L_{s}(\mu \mathrm{H})$ & 6.9997 & 7.1129 & 7.0033 & -1.5915 & -1.5409 \\
\hline$M(\mu \mathrm{H})$ & 6.6808 & 6.7250 & 6.5330 & -0.6572 & -2.8550 \\
\hline$k$ & 0.9544 & 0.9455 & 0.9328 & 0.9413 & -1.3432 \\
\hline$C_{m}(\mathrm{pF})$ & 152.2700 & 156.8510 & 164.3389 & -2.9206 & 4.7739 \\
\hline$f_{1 \mathrm{p}}(\mathrm{MHz})$ & 37.4526 & 35.7500 & 37.0400 & 4.7625 & 3.6084 \\
\hline$f_{1 \mathrm{v}}(\mathrm{MHz})$ & 4.8893 & 4.7879 & 4.7330 & 2.1178 & -1.1466 \\
\hline
\end{tabular}


TABLE X. BPSC-3: COMPARATIVE ANALYSIS

\begin{tabular}{lccccc}
\hline Parameters & Model & Measurement & EM Simulation & Model & EM Simulation \\
\hline$C_{p}(\mathrm{pF})$ & 1.4513 & 1.4650 & 1.4564 & -0.9352 & -0.5870 \\
\hline$L_{s}(\mu \mathrm{H})$ & 7.5795 & 7.8754 & 7.7170 & -3.7573 & -2.0113 \\
\hline$M(\mu \mathrm{H})$ & 7.2545 & 7.2387 & 7.0320 & 0.2183 & -2.8555 \\
\hline$k$ & 0.9571 & 0.9192 & 0.9112 & 4.1232 & -0.8703 \\
\hline$C_{m}(\mathrm{pF})$ & 132.2000 & 130.1400 & 135.9030 & 1.5829 & 4.4283 \\
\hline$f_{1 \mathrm{p}}(\mathrm{MHz})$ & 34.3014 & 33.8250 & 34.3400 & 1.4084 & 1.5225 \\
\hline$f_{1 \mathrm{v}}(\mathrm{MHz})$ & 5.0278 & 5.0188 & 4.9743 & 0.1793 & -0.8867 \\
\hline
\end{tabular}

The error for the modeled $C_{p}$ was smaller than $8 \%$ for the three BPSCs of Table VII. This error is related with the accuracy in the calculation of $C_{o v}$ and $C_{s}$, which in turn depends on the accuracy of the degeneration factor curves and upon the precision of the modeled $C_{m}$, associated with equations (31) and (34).

Regarding the error in the modeled $C_{m}$, it depends not only upon the accuracy during the calculation of elliptic integrals, but also on the accuracy during the calculation of average length $l_{a v}$ that, for simplicity, excluded the first two and the last two tracks of BPSC so that the mutual capacitance could be modeled as the capacitance of a CPW line. The exclusion of these tracks causes an error in the calculation of capacitance $C_{m}$, but this error can be limited to $13 \%$ if $l_{a v} /\left(\right.$ Dout $\left._{b}+\operatorname{Din}_{b}\right) \geq 8$ and designing BPSCs with at least a dozen of turns or $N \geq 10$. Considering that in this paper the three BPSCs were manufactured with $N \geq 20$ and $l_{a v} /\left(\right.$ Dout $\left._{b}+\operatorname{Din}_{b}\right) \geq 18$, this procedure ensured an error of less than $3 \%$ in $C_{m}$ regarding to the measured data.

The parameters $L_{s}, M$ and $k$ had errors smaller than $10 \%$ using equations (6) to (14).

The first resonances (peak $f_{\mathrm{lp}}$ and valley $f_{\mathrm{lv}}$ ) of each BPSC, using equations (4) and (5), were estimated with an error of less than $5 \%$.

Tables XI to XIII show $C_{o v}$ and $C_{s}$ of the BPSC-1, BPSC-2 and BPSC- 3 obtained both by the model and by EM simulation. These tables also show, in the last column, the percentage difference or error of each modeled capacitance regarding the simulated values, where it is observed that the respective errors of those two capacitances for the proposed model are smaller than 5\%. It is important to note that the fact that each monofilar winding have been designed with two underpass tracks has make the $C_{o v}$ capacitance significant, representing more than $30 \%$ of the total parasitic capacitance $C_{p}$ for the three analyzed BPSCs. On the other hand, there was a negligible percentage difference of less than $0.5 \%$ when using any of the two underpass tracks to determine the electrical parameters due to the symmetry of the BPSC. 
Finally, because underpass tracks are essential for connecting the BPSC to the impedance analyzer, such tracks could not be extracted from the manufactured BPSC. Thus, it was not possible to measure $C_{o v}$ and $C_{s}$ which prevents a comparative analysis with the values modeled of $C_{o v}$ and $C_{s}$.

TABLE XI. BPSC-1: $C_{o v}$ and $C_{s}$

\begin{tabular}{llll}
\hline Capacitance (pF) & Model & EM Simulation & Error $(\%)$ \\
\hline$C_{s}$ & 0.7409 & 0.7790 & -4.8909 \\
\hline$C_{o v}$ & 0.4800 & 0.4868 & -1.3969 \\
\hline
\end{tabular}

TABLE XII. BPSC-2: $C_{o v}$ and $C_{s}$

\begin{tabular}{llll}
\hline Capacitance (pF) & Model & EM Simulation & Error $(\%)$ \\
\hline$C_{s}$ & 0.8594 & 0.9010 & -4.6171 \\
\hline$C_{o v}$ & 0.4606 & 0.4630 & -0.5184 \\
\hline
\end{tabular}

TABLE XIII. BPSC-3: $C_{o v}$ and $C_{s}$

\begin{tabular}{llll}
\hline Capacitance (pF) & Model & EM Simulation & Error $(\boldsymbol{\%})$ \\
\hline$C_{s}$ & 0.8784 & 0.8780 & 0.0456 \\
\hline$C_{o v}$ & 0.5729 & 0.5784 & -0.9509 \\
\hline
\end{tabular}

\section{CONCLUSIONS}

In this paper an electrical model for the open square BPSC that covers the first valley and the first resonance peak for future applications as PSR sensor and WPT system was presented. The electrical parameters of the model were determined and a new approach was proposed to calculate mutual capacitances of BPSCs, based on CPW lines. In order to validate the proposed model, three BPSCs on FR-4 substrate and with solder mask were manufactured, tested on the impedance analyzer and also submitted to electromagnetic simulations. Subsequently, tables with the main electrical parameters were produced aiming to establish a comparative analysis between the results obtained with the proposed model, by EM simulation, as well as for the measured values. Finally, the parameters of the model were obtained with errors smaller than $10 \%$ and the first valley and the first resonance peak were determined with errors smaller than 5\%, which showed good agreement with the data obtained in the analyzer impedance and by EM simulation.

\section{REFERENCES}

[1] N. Tesla, "Coil for electro-magnets," U.S. Patent 512340, Jan. 09, 1894.

[2] W.C. Wysock, J.F. Corum, J.M. Hardesty and K.L. Corum, "Who Was The Real Dr. Nikola Tesla? (A Look At His Professional Credentials)," Antenna Measurement Techniques Association, pp. 1-5, Oct. 2001.

[3] C. M. de Miranda, "Equationing and modeling de Tesla's bifilar coil and its proposal as a biotelemetric self-resonant sensor," M.S. dissertation, Dept. Electrical Engineering and Applied Computer Sciences, Federal Univ. of Technology - Paraná (UTFPR), Curitiba, PR, Brazil, 2012 (in portuguese).

[4] A. Massarini and M. K. Kazimierczuk, "Self-capacitance of inductors," IEEE transactions on power electronics, v. 12, n. 4, pp. 671-676, July 1997.

[5] C. M. de Miranda and S. F Pichorim, "The Tesla coil as a self-resonant biotelemetric sensor," in XXIII Brazilian Brazilian Microwave and Optoelectronics Society-SBMO received 14 May 2018; for review 14 May 2018; accepted 20 Jun 2018 Brazilian Society of Electromagnetism-SBMag 
Journal of Microwaves, Optoelectronics and Electromagnetic Applications, Vol. 17, No. 3, September 2018339 DOI: http://dx.doi.org/10.1590/2179-10742018v17i31254

Congress of Biomedical Engineering (CBEB), v. 23, Porto de Galinhas, PE, Brazil, Oct. 2012 (in portuguese).

[6] M. Ghovanloo and G. Lazzi, "Transcutaneous magnetic coupling of power and data," in Wiley Encyclopedia of Biomedical Engineering, M. Akay, Ed. Hoboken, NJ: Wiley, 2006.

[7] C. M. de Miranda and S. F. Pichorim, "A Self-Resonant Two-Coil Wireless Power Transfer System Using Open Bifilar Coils," IEEE Transactions on Circuits and Systems II: Express Briefs, vol. 64, no. 6, pp. 615-619, June 2017.

[8] O. Isik and K.P. Esselle, "Design of monofilar and bifilar Archimedean spiral resonators for metamaterial applications," IET Microwaves Antennas \& Propagation, vol. 3, n. 6, pp. 929-935, Oct. 2009.

[9] S. F Pichorim, V. A Marcis and G. T. Laskoski, "Humidity in sandy soil measured by passive, wireless, and resonant sensor with bifilar coil," in First Latin-American Conference on Bioimpedance - CLABIO, Journal of Physics: Conference Series 407, Joinville, SC, Brazil, Oct. 2012.

[10] D. D. Reis, T. E. Cervi and S. F. Pichorim, "Passive resonant sensor using bifilar coil for moisture measurement on woods," in MOMAG 2014: 16th SBMO - Brazilian Symposium on Microwave and Optoelectronics and 11th CBMag - Brazilian Congress of Electromagnetism, Curitiba, PR, Brazil, pp. 198-203, Sept. 2014. (in portuguese).

[11] S. F. Pichorim, "Passive, wireless, resonant sensor with open bifilar winding," Brazilian Patent Application 102013 008282-1 A2, April 05, 2013, in Revista da Propriedade Industrial, Rio de Janeiro, RJ, Brazil, no. 2320, p. 96, June 23, 2015. (in portuguese).

[12] J. Chen and J. J. Liou, "On-chip spiral inductors for RF applications: An overview," Journal of Semiconductor Technology and Science, v. 4, n. 3, pp. 149-167, Sept. 2004.

[13] S. S. Mohan, M. del Mar Hershenson, S. P. Boyd and T. H. Lee, "Simple accurate expressions for planar spiral inductances," IEEE Journal of Solid-State Circuits, vol. 34, no. 10, pp. 1419-1424, Oct. 1999.

[14] G. Ghione and C. U. Naldi, "Coplanar Waveguides for MMIC Applications: Effect of Upper Shielding, Conductor Backing, Finite-Extent Ground Planes, and Line-to-Line Coupling," IEEE Transactions on Microwave Theory and Techniques, vol. 35, no. 3, pp. 260-267, Mar 1987.

[15] R. N. Simons, "Coplanar Waveguide with Finite-Width Ground Planes," in Coplanar Waveguide Circuits, Components and Systems, New York: John Wiley \& Sons, 2001, pp. 112-126.

[16] C.H. Wu, C.C. Tang and S.I. Liu, "Analysis of on-chip spiral inductors using the distributed capacitance model," IEEE Journal of Solid-State Circuits, vol. 38, n. 6, pp.1040-1044, June 2003.

[17] T. Masuda, A. Kodama, T. Nakamura, N. Shiramizu, S.Wada, T. Hashimoto, and K. Washio, "A simplified distribution parasitic capacitance model for on-chip spiral inductors," in Digest of Papers. 2006 Topical Meeting on Silicon Monolithic Integrated Circuits in RF Systems, San Diego, CA (USA), pp. 111-114, Jan. 2006.

[18] LAB Fit - Curve Fitting Software. Available: http://zeus.df.ufcg.edu.br/labfit

[19] J. Olivo, S. Carrara and G. De Micheli, "Modeling of printed spiral inductors for remote powering of implantable biosensors," in 5th International Symposium on Medical Information and Communication Technology, Montreux, Switzerland, pp. 29-32, Mar. 2011.

[20] MATLAB (Matrix Laboratory) software. Available: https://www.mathworks.com/products/matlab.html

[21] Keysight Advanced Design System (ADS) software. Available: http://www.keysight.com/find/eesof-ads

[22] X.M. Lopez-Fernández, H.B. Ertan and J. Turowski, "Coreless PCB Transformers," in TRANSFORMERS: Analysis, Design and Measurements. Boca Raton, FL, USA: CRC Press, 2013, pp. 537-542. 